\title{
Hypothermic Intraperitoneal Chemotherapy for Gastrointestinal Malignancies-A Relic of the Past or Useful Tool for Today?
}

\author{
Megan Greally and David H Ilson \\ Gastrointestinal Oncology Service, Department of Medicine, Memorial Sloan Kettering Cancer Center, New York, NY, USA
}

\section{Keywords}

Peritoneal carcinomatosis, isolated peritoneal metastases, cytoreductive surgery, hyperthermic intraperitoneal chemotherapy, gastrointestinal malignancies, colorectal cancer, gastric cancer

Disclosures: David H Ilson is a consultant/advisory board member for Taiho, Pieris, Roche, AstraZeneca, Bayer, BristolMyers Squibb, Merck, and Astellas. Megan Greally has no conflicts of interest to declare in relation to this article.

Review Process: Double-blind peer review.

Compliance with Ethics: This article involves a review of literature and does not report on new clinical data, or any studies with human or animal subjects performed by any of the authors.

Authorship: The named authors meet the criteria of the International Committee of Medical Journal Editors for authorship for this manuscript, take responsibility for the integrity of the work as a whole and have given final approval for the version to be published.

Received: April 1, 2019

Accepted: July 10, 2019

Citation: Oncology \& Hematology Review (US). 2019; 15(2):83-6

Corresponding Author: Megan Greally, Gastrointestinal Oncology Service, Department of Medicine, Memorial Sloan Kettering Cancer Center, 300 E. 66 ${ }^{\text {th }}$ Street, New York, NY 10065, USA. E: greallym@mskcc.org

Support: No funding was received in the publication of this article.
Peritoneal carcinomatosis, defined as tumor dissemination in the peritoneal cavity, is a common manifestation of gastrointestinal malignancies. However, isolated peritoneal metastases (IPM) are relatively rare, though appendiceal tumors and peritoneal mesothelioma have a propensity to involve only the peritoneum. Cytoreductive surgery including hyperthermic intraperitoneal chemotherapy (HIPEC), are now considered standard treatment for appendiceal tumors with peritoneal dissemination. ${ }^{1-3}$ However, there is a paucity of robust high-quality data informing the role of cytoreductive surgery and IPC in patients with other gastrointestinal malignancies who have IPM. This is in contrast to ovarian cancer, for which a recent prospective trial suggested a survival benefit with HIPEC in patients with stage III disease. ${ }^{4}$ This may be because patients with ovarian cancer are generally highly chemosensitive, with higher, more durable response rates to first-line therapy than those observed in gastrointestinal malignancies. . $^{5-7}$ Despite this, HIPEC has not been widely adopted in the treatment of ovarian cancer in the United States due to continued uncertainty regarding the benefit of hyperthermia over IPC alone and potential morbidity and prolonged post-operative recovery that could result in a treatment delay or decreased dose intensity.

\section{Treatment of peritoneal carcinomatosis}

Effective treatment options for peritoneal carcinomatosis from gastrointestinal malignancies have historically represented an unmet need. Management of peritoneal carcinomatosis is challenging due its associated high symptom burden and resultant impact on performance status and ability to tolerate systemic therapies. Furthermore, disease extent is often underrepresented on imaging. Peritoneal carcinomatosis has also been associated with a poorer prognosis compared with metastatic disease at other sites. ${ }^{8.9}$ In this context, there has been considerable interest in evaluating the role of cytoreductive surgery with IPC for IPM, and it has been postulated that IPM may represent a regional, rather than a systemic, disease. Original studies by Sugarbaker reported that cytoreductive surgery could be utilized to remove gross disease, resulting in prolongation of survival..$^{1-12}$ It was also hypothesized that delivering IPC to residual small volume gross or microscopic disease may improve outcomes. ${ }^{12}$

\section{Hyperthermic intraperitoneal chemotherapy}

Recognition of the peritoneum as a hypoxic environment through which chemotherapy penetrates poorly, prompted evaluation of HIPEC with the hypothesis that heat ( $41-43^{\circ}$ Celsius) might promote drug penetration. ${ }^{13,14}$ In addition, hyperthermia is cytotoxic to tumor cells through denaturation of proteins and inhibition of nuclear matrix-medicated functions essential to DNA replication, transcription, and repair. ${ }^{15-17}$ It has also been shown to inhibit angiogenesis. ${ }^{18,19}$ Furthermore, early studies observed that significantly higher chemotherapy concentrations in peritoneal fluid versus systemic blood could be achieved.20.21 For example, peak intraperitoneal concentrations of mitomycin have been demonstrated to be approximately 100-fold higher than peak serum levels. ${ }^{21}$ While 
mitomycin is no longer a standard therapy in gastrointestinal malignancies, it is considered an ideal agent for HIPEC for several reasons. Firstly, a high intraperitoneal concentration can be achieved-it is a large-sized molecule that is not rapidly absorbed systemically and it is stable at high temperatures. ${ }^{14}$ Hyperthermia has also been shown to potentiate the cytotoxic effect of both mitomycin $\mathrm{C}$ and oxaliplatin, an active systemic agent in gastrointestinal malignancies that has also been used as an intraperitoneal agent in gastrointestinal malignancies. ${ }^{14,15}$ The synergistic effect of heat and cytotoxic drugs is thought to arise from increased membrane permeability and transport, resulting in increased drug uptake as well as alteration of cellular metabolism and changes in pharmacokinetics and secretion. The limited drug absorption seen with IPC is also attractive, as systemic toxicity is reduced.

Many questions remain regarding appropriate patient selection for cytoreductive surgery and IPC, the additional benefit of IPC over cytoreductive surgery alone, whether hyperthermia is necessary, and if so, for what duration. The optimal drug of choice also remains unclear. Although mitomycin is no longer utilized as systemic therapy in gastrointestinal malignancies it continues to be administered intraperitoneally. Therefore, techniques remain heterogenous between high volume institutions.

Because cytoreductive surgery and HIPEC may be associated with considerable morbidity, a multidisciplinary approach for appropriate patient selection is important and the use of the peritoneal carcinomatosis index (PCl) can play an important role in predicting the possibility of obtaining a complete cytoreduction. Retrospective series' have reported 5-year overall survival (OS) in $>50 \%$ for patients achieving complete cytoreduction compared to a 20\% 5-year OS in patients who undergo suboptimal surgery. ${ }^{22-24}$

\section{Colorectal cancer}

IPM from colorectal cancer (CRC) occur in 2-8\% of patients and appear to be associated with shorter median survival (13-16 months) than patients with isolated disease at other sites.,25,26 Until recently, only one prospective study had evaluated cytoreductive surgery and HIPEC for IPM in CRC. In this study, 105 patients were randomized to 5-fluorouracil/leucovorin alone or cytoreductive surgery and HIPEC with mitomycin C for 90 minutes, followed by 5 -fluorouracil/leucovorin. ${ }^{27}$ Median OS was significantly improved with cytoreductive surgery and HIPEC (22.3 versus 12.6 months; $p=0.032$ ). The post-operative mortality rate was $8 \%$. With advances in systemic therapy, it is unknown if the outcomes reported here are better than the outcomes that could potentially be achieved with use of contemporary chemotherapy regimens. Furthermore, small numbers of patients were accrued, including patients with peritoneal carcinomatosis of appendiceal origin (17\%), who may obtain more benefit from surgical intervention. Long-term follow-up showed that the 5-year disease-free survival rate was $45 \%$ in patients who had surgery and HIPEC with complete cytoreduction followed by systemic therapy versus $<10 \%$ in patients who underwent incomplete cytoreduction at time of surgery and HIPEC or those treated with chemotherapy alone. ${ }^{28}$

Results from the phase III PRODIGE-7 study were recently reported and addressed the specific role of HIPEC in addition to cytoreductive surgery. ${ }^{29}$ Patients with a $\mathrm{PCl}<25$ received chemotherapy before and/or after cytoreductive surgery and were randomized to receive HIPEC with oxaliplatin. There was no difference in median relapse-free survival or OS between the groups. Morbidity was significantly higher in the HIPEC arm. In an unplanned subgroup analysis, patients with a $\mathrm{PCl}$ of 11-15 who underwent HIPEC showed an OS benefit. Criticisms of this study include the accrual of patients with $\mathrm{PCl}$ of $15-25$ and only a 30-minute administration time for oxaliplatin.

Retrospective studies have shown benefit for cytoreductive surgery and HIPEC. Elias et al. compared patients with CRC and IPM treated with contemporary chemotherapy $(n=48)$ to patients who underwent cytoreductive surgery and HIPEC using oxaliplatin $(n=48)$; median survival was 24 versus 63 months $(p<0.05)$, respectively. ${ }^{30}$ Franko et al. reported improved OS in patients who had cytoreductive surgery and HIPEC versus chemotherapy alone, with a median os of 35 and 17 months $(p<0.001)$, respectively. ${ }^{31}$ Both studies included selected patients with asymptomatic peritoneal carcinomatosis and the use of contemporary chemotherapy is reflected in the favorable survival rates in the chemotherapy only arms. Finally, a multicenter study examined outcomes of patients $(n=506)$ treated with cytoreductive surgery plus HIPEC (54\%), early post-operative IPC (EPIC; $24 \%$ ) or both (22\%). Median OS was 19.2 months. Patients who had complete cytoreduction had improved median OS compared to those who did not (32.4 months versus 8.4 months, $p<0.001) .{ }^{32}$

Preliminary results from two randomized studies (PROPHLOCHIP and COLOPEC) evaluating the role of adjuvant HIPEC, in patients considered at high risk of peritoneal recurrence, have recently been presented.33,34 There was no improvement in outcomes with the addition of HIPEC in either study. The ICARuS study (ClinicalTrials.gov identifier: NCT01815359) is an ongoing randomized phase II study evaluating EPIC (with floxuridine) versus HIPEC (with mitomycin C) following optimal cytoreductive surgery for colorectal and appendiceal cancers with IPM. EPIC and HIPEC have not previously been compared prospectively. When delivered intraperitoneally, hepatic extraction of floxuridine (a fluorinated pyrimidine) is high, allowing high doses to be administered with minimal systemic toxicity. However, EPIC can only penetrate $3 \mathrm{~mm}$ of peritoneal tissue, and may not reach all tumor cells. Potential advantages of HIPEC are that it is given before post-operative adhesions develop (which may prevent uniform perfusion of peritoneal surfaces with EPIC) and hyperthermia is recognized to be cytotoxic in itself. ${ }^{15}$ No cytoreductive surgery only arm was included in this study. The ACOSOG-Z6091 trial attempted to evaluate this question but failed to accrue due to patient perception that randomization to an arm without IPC was unacceptable.

Overall, the role of cytoreductive surgery and especially HIPEC remains controversial in CRC as benefit in the contemporary era has not been definitively shown. National Comprehensive Cancer Network (NCCN) guidelines suggest it can be considered in experienced centers for selected patients with limited peritoneal metastases for whom complete cytoreduction can be achieved. The independent contribution of HIPEC over cytoreductive surgery alone remains difficult to assess since they are undertaken synchronously, and recent data, using oxaliplatin, is unconvincing. Improvements in systemic therapies may negate the potential benefit of HIPEC. However, the median OS of approximately 40 months in the PRODIGE-7 study is favorable when compared to historical controls, suggesting that cytoreductive surgery may have a role in selected patients. $\mathrm{PCI}$ has been validated as a prognostic indicator and can be used as a guide for intraoperative decision making. ${ }^{35}$ Upfront chemotherapy may facilitate identification of patients most likely to benefit. Importantly, the mortality risk of cytoreductive surgery and HIPEC has fallen in contemporary studies with rates of $1.1-4.0 \%$ reported. ${ }^{32,36,37}$ With improved understanding regarding 
patient selection, cytoreductive surgery remains reasonable in high volume centers. However, the additional benefit of HIPEC is questionable.

\section{Gastric cancer}

The role of cytoreductive surgery and HIPEC in patients with gastric cancer and IPM remains very unclear. Studies from East Asia, mostly retrospective, suggest a benefit for HIPEC. ${ }^{38-40}$ In 2011, Yang et al. reported the results of a randomized phase III trial of almost 70 patients, comparing cytoreductive surgery alone to cytoreductive surgery plus HIPEC. An OS benefit was observed in the patients who underwent HIPEC compared to those who underwent cytoreductive surgery alone (11 versus 6.5 months; $p<0.05$ ). ${ }^{41}$ The GYMSSA trial also showed an improvement in median OS in patients who underwent cytoreductive surgery plus HIPEC versus patients who received chemotherapy (11.3 versus 4.3 months). ${ }^{42}$ Patients in the surgery arm who survived $>1$ year all had complete cytoreduction. However, this study enrolled only 17 patients and is considered hypothesis-generating at best. Results of a retrospective French study, CYTO-CHIP, reported outcomes of 180 patients who underwent cytoreductive surgery and HIPEC compared to 97 patients who underwent cytoreductive surgery alone. ${ }^{43} \mathrm{PCl}$ was higher in the HIPEC arm ( 6 versus $2, \mathrm{p}=0.003$ ) and there was no difference in completeness of cytoreductive surgery between groups. HIPEC was associated with improved OS (median, 18.8 versus 12.1 months; 5-year OS, $20 \%$ versus 6\%). Morbidity and mortality were similar between groups.

The use of HIPEC in gastric cancer remains experimental in light of conflicting results from underpowered studies and absence of high-level data. NCCN guidelines suggest that gastric cancers should be considered unresectable with any evidence of peritoneal involvement including positive peritoneal cytology. Of note, one retrospective study suggested that patients with positive peritoneal cytology who have cleared cytology after a period of chemotherapy have improved disease-specific survival compared to patients who do not. ${ }^{44}$ Furthermore, several studies have shown that patients who undergo complete/near complete resection have improved survival over patients who undergo incomplete resection. ${ }^{41,45,46}$ Therefore, there may be a role for HIPEC in highly selected patients with positive peritoneal cytology or minimal gross disease that is diagnosed using laparoscopy but is not visible on imaging. Patients who manifest chemosensitive disease through resolution of gross disease and/or positive peritoneal cytology may be candidates for gastrectomy and HIPEC, hypothesizing that patients with disease sensitive to chemotherapy may be more sensitive to HIPEC. While survival may be extended over chemotherapy alone, it is unlikely that patients can be cured with this approach. A standardized multimodal approach to this group of patients remains an unmet need. If considered, surgery and HIPEC should only be undertaken in specialized centers.

\section{Other gastrointestinal malignancies}

There is limited data regarding the role of cytoreductive surgery and HIPEC in other gastrointestinal malignancies. Peritoneal mesothelioma is a rare malignancy characterized by progression restricted to the peritoneum. Several studies have reported a median survival of 3-5 years with cytoreductive surgery and HIPEC which compares favorably to historical studies reporting survival without surgery of approximately 1 year. ${ }^{47}$ Patient selection is key and surgical intervention should be undertaken only in experienced centers. Data regarding the merits of an aggressive surgical approach in patients with hepatobiliary and pancreas cancers is extremely limited. Two small retrospective studies suggested improved survival outcomes in small cohorts of patients with biliary and hepatocellular cancers respectively. ${ }^{48,49}$ No conclusions can be drawn due to study size and lack of prospective data.

\section{Conclusion}

The benefit of cytoreductive surgery with or without HIPEC in gastrointestinal malignancies may never be clearly defined. The shortcomings of retrospective studies combined with limited prospective randomized data means there is little to guide management of this patient subgroup, especially in non-CRC/appendiceal malignancies. Large informative trials are not feasible in a Western population in gastric cancer and in CRC it is difficult to accrue patients to studies in which they forego treatment with surgery or HIPEC.

It is likely that there is a small subset of patients whose disease does not have a propensity to metastasize to other sites, on a spectrum ranging from appendiceal neoplasms and peritoneal mesothelioma to gastric, colorectal and hepatobiliary-pancreatic cancers. Future directions may involve determining, through molecular genetic profiling, which patients are likely to have disease confined to the peritoneal cavity and those destined to progress at other sites. Improved detection techniques would also help improve our knowledge of disease extent and biology. In the right patients, it is likely that cytoreductive surgery with or without HIPEC can improve outcomes and sometimes result in long-term remission. •
1. Lambert LA. Looking up: Recent advances in understanding and treating peritoneal carcinomatosis. CA Cancer J Clin. 2015;65:284-98

2. Gonzalez-Moreno S. Peritoneal Surface Oncology: A progress report. Eur J Surg Oncol. 2006;32:593-6.

3. Chua TC, Moran BJ, Sugarbaker PH, et al. Early- and long-term outcome data of patients with pseudomyxoma peritonei from appendiceal origin treated by a strategy of cytoreductive surgery and hyperthermic intraperitoneal chemotherapy. I Clin Oncol. 2012:30:2449-56.

4. van Driel WJ, Koole SN, Sikorska K, et al. Hyperthermic Intraperitoneal Chemotherapy in Ovarian Cancer. N Engl J Med. 2018;378:230-240.

5. Katsumata N, Yasuda M, Takahashi F, et al. Dose-dense paclitaxe once a week in combination with carboplatin every 3 weeks for advanced ovarian cancer: a phase 3, open-label, randomised controlled trial. Lancet. 2009:374:1331-8.

6. Pignata S, Scambia G, Ferrandina G, et al. Carboplatin plus paclitaxel versus carboplatin plus pegylated liposomal doxorubicin as first-line treatment for patients with ovarian cancer: the MITO-2 randomized phase III trial. J Clin Oncol. 2011;29:3628-35

7. Pignata S, Scambia G, Katsaros D. Carboplatin plus paclitaxel once a week versus every 3 weeks in patients with advanced ovarian cancer (MITO-7): a randomised, multicentre, open-label, phase 3 trial. Lancet Oncol. 2014;15:396-405

8. Franko J, Shi Q, Goldman CD, et al. Treatment of colorecta peritoneal carcinomatosis with systemic chemotherapy: a pooled analysis of north central cancer treatment group phase III trials N9741 and N9841. J Clin Oncol. 2012;30:263-7.

9. Thomassen I, van Gestel YR, van Ramshorst B, et al. Peritoneal carcinomatosis of gastric origin: a population-based study on incidence, survival and risk factors. Int I Cancer. 2014;134:622-8.

10. Sugarbaker PH, Zhu BW, Banez Sese G, et al. Peritoneal carcinomatosis from appendiceal cancer: results in 69 patients treated by cytoreductive surgery and intraperitoneal Chemotherapy. Dis Colon Rectum. 1993;36:323-9.

11. Sugarbaker PH, Jablonski KA. Prognostic features of 51 colorectal and 130 appendiceal cancer patients with peritoneal carcinomatosis treated by cytoreductive surgery and intraperitoneal Chemotherapy. Ann Surg. 1995:221:124-32.

12. Sugarbaker PH. Peritonectomy procedures. Ann Surg. 1995:221:29-42.

13. Dedrick RL, Flessner MF. Pharmacokinetic problems in peritoneal drug administration: tissue penetration and surface exposure. JNCl. 1997:89:480-7.

14. Goodman MD, MCPartland S, Detelich D, et al. Chemotherapy for intraperitoneal use: a review of hyperthermic intraperitoneal chemotherapy and early post-operative intraperitoneal chemotherapy. J Gastrointest Oncol. 2016;7:45-57.
15. Gonzalez-Moreno S, Gonzalez-Bayon LA Ortega-Perez G Hyperthermic intraperitoneal chemotherapy: Rationale and technique. World I Gastrointest Oncol. 2010;2:68-75.

16. Ohno S, Siddik ZH, Kido Y, et al. Thermal enhancement of drug uptake and DNA adducts as a possible mechanism for the effect of sequencing hyperthermia on cisplatin-induced cytotoxicity in L1210 cells. Cancer Chemother Pharmacol. 1994;34:302-6.

17. Hildebrandt B, Wust P, Ahlers $\mathrm{O}$, et al. The cellular and molecular basis of hyperthermia. Crit Rev Oncol Hematol. 2002;43:33-56.

18. Sawaji Y, Sato T, Takeuchi A, et al. Anti-angiogenic action of hyperthermia by suppressing gene expression and production of tumour-derived vascular endothelial growth factor in vivo and in vitro. Br J Cancer. 2002;86:1597-603.

19. Roca C, Primo L, Valdembri D, et al. Hyperthermia inhibits angiogenesis by a plasminogen activator inhibitor 1-dependent mechanism. Cancer Res. 2003;63:1500-7.

20. Sugarbaker PH, Graves T, DeBruijn EA, et al. Early postoperative intraperitoneal chemotherapy as an adjuvant therapy to surgery for peritoneal carcinomatosis from gastrointestinal cancer: pharmacological studies. Cancer Res. 1990;50:5790-4.

21. Shen P, Hawksworth J, Lovato J, et al. Cytoreductive surgery and intraperitoneal hyperthermic chemotherapy with mitomycin C for peritoneal carcinomatosis from nonappendiceal colorectal carcinoma. Ann Surg Oncol. 2004;11:178-86.

22. Culliford AT, Brooks AD, Sharma S, et al. Surgical debulking 
and intraperitoneal chemotherapy for established peritoneal metastases from colon and appendix cancer. Ann Surg Oncol. 2001;:8787-95.

23. Sugarbaker PH. Cytoreductive surgery and peri-operative intraperitoneal chemotherapy as a curative approach to pseudomyxoma peritonei syndrome. Eur I Surg Oncol. 2001;27:239-43.

24. Miner TJ, Shia J, Jaques DP, et al. Long-term survival following treatment of pseudomyxoma peritonei: an analysis of surgical therapy. Ann Surg. 2005;241:300-8.

25. Franko J, Shi Q, Meyers JP, et al. Prognosis of patients with peritoneal metastatic colorectal cancer given systemic therapy: an analysis of individual patient data from prospective randomised trials from the Analysis and Research in Cancers of the Digestive System (ARCAD) database Lancet Oncol. 2016;17:1709-19.

26. Segelman J, Granath F, Holm T, et al. Incidence, prevalence and risk factors for peritoneal carcinomatosis from colorectal cance. Br J Surg. 2012;99:699-705.

27. Verwaal VJ, van Ruth S, de Bree E, et al. Randomized trial of cytoreduction and hyperthermic intraperitoneal chemotherapy versus systemic chemotherapy and palliative surgery in patients with peritoneal carcinomatosis of colorectal cancer. J Clin Oncol. 2003;21:3737-43

28. Verwaal VJ, Bruin S, Boot H, et al. 8-year follow-up of randomized trial: cytoreduction and hyperthermic intraperitoneal chemotherapy versus systemic chemotherapy in patients with peritoneal carcinomatosis of colorectal cancer. Ann Surg Oncol. 2008;15:2426-32.

29. Quenet F, Elias D, Roca $L$, et al. A UNICANCER phase III trial of Hyperthermic Intra-peritoneal Chemotherapy (HIPEC) for Colorectal Peritoneal Carcinomatosis. PRODIGE 7. Eur J surg Oncol. 2019;45:e17.

30. Elias D, Lefevre JH, Chevalier J, et al. Complete cytoreductive surgery plus intraperitoneal chemohyperthermia with oxaliplatin for peritoneal carcinomatosis of colorectal origin. J Clin Oncol. 2009;27:681-5.

31. Franko J, Ibrahim Z, Gusani NJ, et al. Cytoreductive surgery and hyperthermic intraperitoneal chemoperfusion versus systemic chemotherapy alone for colorectal peritoneal carcinomatosis.
Cancer. 2010;116:3756-62.

32. Glehen O, Kwiatkowski F, Sugarbaker PH, et al. Cytoreductive surgery combined with perioperative intraperitoneal chemotherapy for the management of peritoneal carcinomatosis from colorectal cancer: a multi-institutional study. I Clin Oncol. 2004;22:3284-92.

33. Goere $\mathrm{D}$, Glehen $\mathrm{O}$, Quenet $\mathrm{F}$, et al. Results of a randomized phase 3 study evaluating the potential benefit of a secondlook surgery plus HIPEC in patients at high risk of developing colorectal peritoneal metastases (PROPHYLOCHIPNTC01226394). Presented at the Annual Society of Clinical Oncology, Chicago, June 3rd 2018.

34. Klaver CEL, Wisselink DD, Punt CJA, et al. Adjuvant HIPEC in patients with colon cancer at high risk of peritoneal metastases: Primary outcome of the COLOPEC multicenter randomized trial. Presented at the Gastrointestinal Cancers Symposium, trial. Presented at the Gastrointesting
San Francisco, January 19, 2019.

35. Faron M, Macovei R, Goere D, et al. Linear Relationship of Peritoneal Cancer Index and Survival in Patients with Peritoneal Metastases from Colorectal Cancer. Ann Surg Oncol. 2016;23:114-9.

36. Foster JM, Sleightholm R, Patel A, et al. Morbidity and Mortality Rates Following Cytoreductive Surgery Combined With Hyperthermic Intraperitoneal Chemotherapy Compared With Other High-Risk Surgical Oncology Procedures. JAMA Netw Open. 2019;2:e186847.

37. Elias D, Gilly F, Boutitie F, et al. Peritoneal colorectal carcinomatosis treated with surgery and perioperative intraperitoneal chemotherapy: retrospective analysis of 523 patients from a multicentric French study. J Clin Oncol. 2010;28:63-8.

38. Koga S, Hamazoe R, Maeta M, et al. Prophylactic therapy for peritoneal recurrence of gastric cancer by continuous hyperthermic peritoneal perfusion with mitomycin C. Cancer. 1988;61:232-7.

39. Yonemura Y, Fujimura T, Fushida S, et al. Hyperthermochemotherapy combined with cytoreductive surgery for the treatment of gastric cancer with peritoneal dissemination. World I Surg. 1991;15:530-5; discussion 535-6.

40. Fujimura T, Yonemura Y, Muraoka K, et al. Continuous hyperthermic peritoneal perfusion for the prevention of peritoneal recurrence of gastric cancer: randomized controlled study. World J Surg. 1994;18:150-5

41. Yang XJ, Huang CQ, Suo T, et al. Cytoreductive surgery and hyperthermic intraperitoneal chemotherapy improves survival of patients with peritoneal carcinomatosis from gastric cancer: final results of a phase III randomized clinical trial. Ann Surg Oncol. 2011:18:1575-81.

42. Rudloff $\mathrm{U}$, Langan RC, Mullinax JE, et al. Impact of maxima cytoreductive surgery plus regional heated intraperitoneal chemotherapy (HIPEC) on outcome of patients with peritoneal carcinomatosis of gastric origin: results of the GYMSSA trial. J Surg Oncol. 2014;110:275-84.

43. Bonnot P-E, Piessen G, Kepenekian V, et al. Cytoreductive Surgery With or Without Hyperthermic Intraperitoneal Chemotherapy fo Gastric Cancer With Peritoneal Metastases (CYTO-CHIP study): A Propensity Score Analysis. J Clin Oncol. 2019;37:2028-40.

44. Mezhir JJ, Shah MA, Jacks LM, et al. Positive peritoneal cytology in patients with gastric cancer: natural history and outcome of 291 patients. Ann Surg Oncol. 2010;17:3173-80.

45. Glehen O, Gilly FN, Arvieux C, et al. Peritoneal carcinomatosis from gastric cancer: a multi-institutional study of 159 patients treated by cytoreductive surgery combined with perioperative intraperitoneal chemotherapy. Ann Surg Oncol. 2010;17:2370-7.

46. Hall JJ, Loggie BW, Shen P, et al. Cytoreductive surgery with intraperitoneal hyperthermic chemotherapy for advanced gastric cancer. J Gastrointest Surg. 2004;8:454-63.

47. Sugarbaker PH, Turaga KK, Alexander HR, Jr., et al. Management of Malignant Peritoneal Mesothelioma Using Cytoreductive Surgery and Perioperative Chemotherapy 1 Oncol Pract. 2016;12:928-35

48. Amblard I, Mercier F, Bartlett DL, et al. Cytoreductive surgery and HIPEC improve survival compared to palliative chemotherapy for biliary carcinoma with peritoneal metastasis: A multi-institutiona cohort from PSOGI and BIG RENAPE groups. Eur I Surg Oncol. 2018;44:1378-83

49. Tabrizian P, Franssen B, Jibara G, et al. Cytoreductive surgery with or without hyperthermic intraperitoneal chemotherapy in patients with peritoneal hepatocellular carcinoma. I Surg Oncol. 2014;110:786-90. 